ORIGINAL ARTICLE

\title{
Epidemiologic Survey of Dental Caries Prevalence and Oral Hygiene Status in 3-20-year-old Young Thalassemia Major Children of Lahore, Pakistan
}

\author{
ALI ANWAAR ${ }^{1}$, ARHAM NAWAZ CHOHAN², MUHAMMAD SHAIRAZ SADIQ ${ }^{3}$, FAREED AHMED ${ }^{4}$, ADEEL IJAZ RANA $^{5}$, \\ BILAL ABDUL QAYUM MIRZA 6 \\ ${ }^{1}$ Assistant Professor, Department of Community \& Preventive Dentistry, Institute of Dentistry, CMH Lahore Medical College, Lahore, \\ Pakistan. National University of Medical Sciences. Pakistan \\ ${ }^{2}$ Professor, Department of Paediatric Dentistry, Institute of Dentistry, CMH Lahore Medical College, Lahore, Pakistan. National University of \\ Medical Sciences. Pakistan \\ ${ }^{3}$ Associate Professor, Department of Oral Medicine, Institute of Dentistry, CMH Lahore Medical College, Lahore, Pakistan. National \\ University of Medical Sciences. Pakistan \\ ${ }^{4}$ Assistant Professor \& HOD, Department of Oral Medicine, Institute of Dentistry, CMH Lahore Medical College, Lahore, Pakistan. National \\ University of Medical Sciences. Pakistan \\ ${ }^{5}$ Demonstrator, Department of Community \& Preventive Dentistry, Institute of Dentistry, CMH Lahore Medical College, Lahore, Pakistan. \\ National University of Medical Sciences. Pakistan \\ ${ }^{6}$ Professor \& HOD, Department of Community \& Preventive Dentistry, Institute of Dentistry, CMH Lahore Medical College, Lahore, Pakistan. \\ National University of Medical Sciences. Pakistan \\ Correspondence to Dr Ali Anwaar, ali_anwaar@yahoo.com; +92 3004212706
}

\begin{abstract}
Background \& Objectives: A Cross-sectional study aimed to assess the prevalence of dental caries and oral hygiene status in Thalassemia Major children age ranging in 3-20-year-old undergoing blood transfusion from the Thalassemia Society of Pakistan. Oral hygiene status and dmft scores were compared.

Methods \& Materials: A total of 117 children from the Thalassemia Society of Pakistan were checked. Three stations were setup for dental check-up and examiners were calibrated. Examination was performed on children sitting comfortability in chairs using sterilised dental mirrors and probes under natural sunlight on modifies version of World Health Organization (WHO) assessment form. Decayed, missing, and filled teeth using DMFT and dmft indices and Oral Hygiene status was recorded as good, fair, and poor.

Results: It was found in our study, 73 males (62.4\%) and 44 females (37.6 \%). mean dmft score for male was $2.25+2.87$. Female score was $2.64 \pm 3.26$. Overall mean DMFT score $2.39 \pm 3.02$.A strong association was observed between age and DMFT $(\mathrm{p}=0.001)$ between Out of the total sample size, two third majority had fair oral hygiene, while the remainder fell into good and poor hygiene category, respectively.

Conclusion: Majority of sample population had fair oral hygiene and young age group were more prone to dental caries.

Keywords: Thalassemia Major, Dental Caries, Oral Health
\end{abstract}

\section{INTRODUCTION}

The Haemoglobinopathies which constitutes Thalassemia and Sickle-cell anaemia have a worldwide spread accounting for $5 \%$ of the overall world population ${ }^{1}$. The worldwide carrier (inheritance in individuals with one mutant gene from one parent) of Thalassemia are higher than Sickle Cell Anemia. ${ }^{1,2}$ Sickle cell disease (SCD) and thalassemia, both are autosomal inherited recessive blood diseases. 2,3 Most commonly found in Tropic and Subtropics region of the world, though migration has made it prevalent worldwide ${ }^{2,3}$

In Pakistan, thalassemia is one of the most common inherited diseases. ${ }^{4}$ Approximately 9000 children are born with Beta Thalassemia every year which can be attributed to consanguineous or cousin marriages ${ }^{4,5,6}$. The estimated burden of the disease is $5-7 \%$ with approximately 9.8 million population suffering from this disease in Pakistan. ${ }^{4}$ The downside in Pakistan is that there is no concept or idea of premarital screening for Thalassemia or counselling of individuals who opt for cousin marriage..$^{5,6}$

Due to the oral structural and physiological changes that occur in Thalassemia major patients, their oral hygiene in general is found to be poor. ${ }^{7-8}$ Moreover, usually the focus is on the treatment of their life-threatening condition instead of treating their dental health. ${ }^{9}$. This study aimed to assess the dental caries and oral hygiene status of beta thalassemia major patients aged between 6 to 20 years in the Thalassemic Institute of Lahore, Pakistan. In this region, limited research work has been done on the oral health status and dental caries experience of Thalassemia patients.

\section{METHODS AND MATERIALS}

A convenient sample of 117 children from the Thalassemia Society of Pakistan were checked. The children ranged between 6-20 years age group. All examiners were given prior training to reduce inter-examiner bias. Examination was performed using dental mirror under natural sunlight on the World Health Organization (WHO) Assessment Form 2013. DMFT/dmft indices and Oral Hygiene status was recorded as good, fair, and poor. Univariate analysis was performed (Mean, frequencies) Data analysis was performed on SPSS (23). ${ }^{10}$ For bivariate analysis, Chisquare was performed for comparison among age and $\mathrm{dmft}$, DMFT and oral health status. For all tests, level of significance was set at $p<0.05$ at confidence level 95\%. Inclusion Criteria: Thalassemic patient with/ without any systemic or dental disease. 
Exclusion Criteria: Patients suffering from other diseases e.g. Diabetes Mellitus or Down's syndrome.

\section{RESULTS}

The $(\boldsymbol{n}=117)$ yielded demographic data of male and females to be $62.4 \%$ (73) and $37.6 \%$ (44). The oral health status of male and females are depicted in Table 1. Fair category comprised of maximum number of study participants in both genders with $56.2 \%$ and $61.4 \%$ respectively followed by good and poor category.

Mean age, mean D, M, F, mean DMFT and dmft (primary and permanent dentition indices) have been depicted in table 2. Mean age was $10.34 \pm 4.81$. Mean values of $D, M, F$ were $2.5 \pm 2.89,0.17 \pm 0.69$ and $0.06 \pm$ 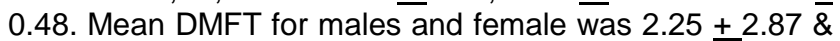 $2.64+3.26$. Mean dmft values of males and female were $2.82 \pm 3.34 \& 2.64 \pm 3.46$, respectively.

The bivariate aspect of the study has been depicted in table 3 with frequency of age and DMFT revealed a strong association between age and DMFT. $(p=0.001)$. There was no association between age and dft (Primary dentition), gender and DMFT/dmft nor was there any association observed in oral health status and $\mathrm{dmft}$.

The distribution of $\mathrm{dmft}$ amongst primary dentition with age yielded the maximum number of decayed, missing or treated teeth was at the age of 7 years with $15 \mathrm{dmft}$ score. The next score observed was at the age of 4 and 6 in the primary dentition was a tie at 12 . Scores greater than 10 were mostly observed in the primary and mixed dentition owing to the socioeconomic condition of the child.

\section{DISCUSSION}

The present study was an effort to identify the oral health problems of the children suffering from $\beta$-Thalassemia major in Lahore, Pakistan. Our study results pertaining to oral health status were comparable in all categories especially 'Fair' with the results of the study conducted in Lahore however there was a comparison group of normal individuals with thalassemia patients. ${ }^{11}$ This need of care can be attributed to disregard on the portion of caregivers conjointly to budgetary status of these families who are as of now burdened with other life threatening Thalassemic condition. ${ }^{11}$

The component of ' $D$ ' from the results showed maximum number was observed in both categories of primary \& permanent dentition. Furthermore, M component was (missing due to caries) was the second among the results. The mean DMFT and dmft score were comparable between the young age and adolescents/young adults. These results were comparable with a study conducted in Northern Thailand. ${ }^{12}$ This may be linked with socioeconomic status of the individual or the family, moreover, the educational background may also be attritubed. ${ }^{13,15}$.

The dmft score and age distribution depicted the same results as the permanent counterparts citing the level of the individual/family of the child in terms of socioeconomic background. ${ }^{13,15}$.

No association was observed between dmft/DMFT and oral health or gender however, a strong association was seen between age and DMFT $(p=0.001)$. These results are comparable with another study conducted in India for the DMFT score however their study sample was six-fold compared to our study with gingival, plaque and control group ${ }^{14}$. When compared with another study conducted in Lahore Pakistan, it yielded similar results with dmft score and age group ${ }^{11}$, fortunately, their sample size was comparable to our study group. ${ }^{11}$

\section{CONCLUSION}

Special instructions in oral hygiene and dental caries prevention are considered an essential in Thalassemia patients to prevent future dental disease and disease progression. It is recommended that whilst these children visit to the transfusion centres, they should be provided with oral hygiene instructions/ education. Moreover, also receive regular dental treatment and follow-up care.

Conflict of Interest: The authors had no conflict of interest. The authors whose names are mentioned in the manuscript certify they have NO affiliations with or involvement in any organization or affiliation with any financial interest (educational grant, participation as speaker, consultancies, organization membership). The authors also certify that the manuscript in question has not been published in any local or international journal or newspaper and the matter or material discussed in the manuscript is not in collaboration with any individual or organization.

Ethical Approval: Ethical Review Board, $\mathrm{CMH}$ Lahore Medical College \& Institute of Dentistry granted the approval. ( ) The Thalassaemic society of Pakistan and the parents of the children granted written consent for conducting the examination/study.

Funding of the Study: The author bears all the expenses conducting the research. No funding was provided from the any institute or any organisation.

Table 1 illustrates the oral health status amongst male and female students.
\begin{tabular}{|l|l|l|l|l|}
\hline $\begin{array}{l}\text { Oral } \\
\text { Health }\end{array}$ & Males & Females \\
\cline { 2 - 5 } & Frequency & Percentage & Frequency & Percentage \\
\hline Good & 15 & $20.5 \%$ & 12 & $27.3 \%$ \\
\hline fair & 41 & $56.2 \%$ & 27 & $61.4 \%$ \\
\hline Poor & 17 & $23.3 \%$ & 5 & $11.4 \%$ \\
\hline total & 73 & $100.0 \%$ & 44 & $100.0 \%$ \\
\hline
\end{tabular}

Table 2 depicting mean score for each category.

\begin{tabular}{|l|l|}
\hline Mean \pm SD \\
\hline Mean Age & $10.34 \pm 4.81$ \\
\hline Mean D & $2.5 \pm 2.89$ \\
\hline Mean M & $0.17 \pm 0.69$ \\
\hline Mean F & $0.06 \pm 0.48$ \\
\hline Mean DMFT & $2.39 \pm 3.02$ \\
\hline Mean dmft & $2.75 \pm 3.37$ \\
\hline
\end{tabular}



Bar graph showing frequency of DMFT with Age. 
Table 3: Distribution of DMFT with age

\begin{tabular}{|c|c|c|c|c|c|c|c|c|c|c|c|c|c|}
\hline \multirow[b]{2}{*}{ Age } & \multicolumn{12}{|c|}{ DMFT } & \multirow[t]{2}{*}{ Total } \\
\hline & 0 & 1 & 2 & 3 & 4 & 5 & 6 & 7 & 8 & 9 & 12 & 13 & \\
\hline 3 & 3 & 0 & 0 & 0 & 0 & 0 & 0 & 0 & 0 & 0 & 0 & 0 & 3 \\
\hline 4 & 12 & 0 & 0 & 0 & 0 & 0 & 0 & 0 & 0 & 0 & 0 & 0 & 12 \\
\hline 5 & 3 & 0 & 1 & 0 & 0 & 0 & 0 & 0 & 0 & 0 & 0 & 0 & 4 \\
\hline 6 & 11 & 0 & 0 & 0 & 1 & 0 & 0 & 0 & 0 & 0 & 0 & 0 & 12 \\
\hline 7 & 7 & 2 & 4 & 1 & 1 & 0 & 0 & 0 & 0 & 0 & 0 & 0 & 15 \\
\hline 8 & 2 & 0 & 1 & 0 & 0 & 0 & 0 & 0 & 0 & 0 & 0 & 0 & 3 \\
\hline 9 & 1 & 1 & 0 & 0 & 0 & 1 & 0 & 0 & 0 & 0 & 0 & 0 & 3 \\
\hline 10 & 3 & 1 & 1 & 1 & 1 & 0 & 0 & 0 & 0 & 0 & 1 & 0 & 8 \\
\hline 11 & 1 & 2 & 1 & 2 & 2 & 0 & 0 & 0 & 0 & 0 & 0 & 0 & 8 \\
\hline 12 & 3 & 2 & 2 & 0 & 4 & 0 & 0 & 0 & 2 & 1 & 0 & 0 & 14 \\
\hline 13 & 1 & 1 & 1 & 1 & 1 & 0 & 0 & 1 & 1 & 0 & 0 & 0 & 7 \\
\hline 14 & 1 & 1 & 1 & 0 & 0 & 0 & 0 & 2 & 0 & 0 & 0 & 0 & 5 \\
\hline 15 & 0 & 1 & 0 & 0 & 0 & 3 & 0 & 1 & 0 & 1 & 0 & 0 & 6 \\
\hline 16 & 1 & 1 & 0 & 0 & 0 & 0 & 0 & 1 & 0 & 0 & 0 & 0 & 3 \\
\hline 18 & 0 & 0 & 0 & 0 & 1 & 0 & 1 & 1 & 0 & 0 & 0 & 0 & 3 \\
\hline 19 & 0 & 1 & 0 & 0 & 1 & 0 & 0 & 0 & 0 & 2 & 0 & 0 & 4 \\
\hline 20 & 1 & 0 & 0 & 1 & 2 & 0 & 0 & 1 & 0 & 1 & 0 & 1 & 7 \\
\hline & 50 & 13 & 12 & 6 & 14 & 4 & 1 & 7 & 3 & 5 & 1 & 1 & 117 \\
\hline
\end{tabular}

Table 4: Distribution of $\mathrm{dft}$ with age.

\begin{tabular}{|c|c|c|c|c|c|c|c|c|c|c|c|c|c|c|}
\hline . & $\mathrm{dmft}$ & & & & & & & & & & & & & \\
\hline Age & 0 & 1 & 2 & 3 & 4 & 5 & 6 & 7 & 8 & 9 & 10 & 11 & 15 & Total \\
\hline 3 & 1 & 0 & 1 & 1 & 0 & 0 & 0 & 0 & 0 & 0 & 0 & 0 & 0 & 3 \\
\hline 4 & 2 & 2 & 0 & 1 & 0 & 0 & 3 & 2 & 0 & 1 & 1 & 0 & 0 & 12 \\
\hline 5 & 1 & 1 & 0 & 0 & 0 & 1 & 0 & 0 & 1 & 0 & 0 & 0 & 0 & 4 \\
\hline 6 & 3 & 0 & 1 & 2 & 1 & 0 & 1 & 2 & 0 & 1 & 1 & 0 & 0 & 12 \\
\hline 7 & 1 & 0 & 1 & 1 & 1 & 2 & 2 & 1 & 3 & 1 & 1 & 0 & 1 & 15 \\
\hline 8 & 1 & 1 & 0 & 0 & 0 & 0 & 0 & 1 & 0 & 0 & 0 & 0 & 0 & 3 \\
\hline 9 & 0 & 0 & 0 & 0 & 2 & 0 & 1 & 0 & 0 & 0 & 0 & 0 & 0 & 3 \\
\hline 10 & 1 & 1 & 0 & 1 & 0 & 1 & 1 & 2 & 0 & 0 & 0 & 1 & 0 & 8 \\
\hline 11 & 2 & 1 & 4 & 0 & 0 & 0 & 1 & 0 & 0 & 0 & 0 & 0 & 0 & 8 \\
\hline 12 & 7 & 3 & 1 & 0 & 0 & 2 & 1 & 0 & 0 & 0 & 0 & 0 & 0 & 14 \\
\hline 13 & 6 & 0 & 1 & 0 & 0 & 0 & 0 & 0 & 0 & 0 & 0 & 0 & 0 & 7 \\
\hline 14 & 5 & 0 & 0 & 0 & 0 & 0 & 0 & 0 & 0 & 0 & 0 & 0 & 0 & 5 \\
\hline 15 & 6 & 0 & 0 & 0 & 0 & 0 & 0 & 0 & 0 & 0 & 0 & 0 & 0 & 6 \\
\hline 16 & 3 & 0 & 0 & 0 & 0 & 0 & 0 & 0 & 0 & 0 & 0 & 0 & 0 & 3 \\
\hline 18 & 3 & 0 & 0 & 0 & 0 & 0 & 0 & 0 & 0 & 0 & 0 & 0 & 0 & 3 \\
\hline 19 & 4 & 0 & 0 & 0 & 0 & 0 & 0 & 0 & 0 & 0 & 0 & 0 & 0 & 4 \\
\hline 20 & 7 & 0 & 0 & 0 & 0 & 0 & 0 & 0 & 0 & 0 & 0 & 0 & 0 & 7 \\
\hline Total & 53 & 9 & 9 & 6 & 4 & 6 & 10 & 8 & 4 & 3 & 3 & 1 & 1 & 117 \\
\hline
\end{tabular}

\section{REFERENCES}

1. Viprakasit V, Ekwattanakit S. Clinical Classification, Screening and Diagnosis for Thalassemia. Hematol Oncol Clin North Am. 2018 Apr;32(2):193-211. doi: 10.1016/j.hoc.2017.11.006. PMID: 2945872

2. Yacobovich $\mathrm{J}$, Tamary $\mathrm{H}$. Thalassemia major and sickle cell disease in adolescents and young adults. Acta Haematol. 2014;132(3-4):3407. doi: 10.1159/000360235. Epub 2014 Sep 10. PMID: 25228560.

3. Masilamani V, Al Salhi MS, Devanesan S, Algahtani FH, Abu-Salah KM, Ahamad I, Agastian P. Spectral detection of sickle cell anemia and thalassemia. Photodiagnosis Photodyn Ther. 2013 Dec;10(4):429-33. doi: 10.1016/j.pdpdt.2013.04.001. Epub 2013 Jun 13. Erratum in: Photodiagnosis Photodyn Ther. 2019 Sep; 27:490. PMID: 24284096.

4. Maheen H, Malik F, Siddique B, Qidwai A. Assessing Parental Knowledge About Thalassemia in a Thalassemia Center of Karachi, Pakistan. J Genet Couns. 2015 Dec;24(6):945-51. doi: 10.1007/s10897-015-9830-z. Epub 2015 Apr 7. PMID: 25843562.

5. Rahman MU, Lodhi Y. Prospects, and future of conservative management of beta thalassemia major in a developing country. Pak J Med Sci 2004; 20:105-12.

6. Hassan K, Aslam M, Ikram N. Parental knowledge and awareness in cases of thalassemia major. J Pak Inst Med Sci 2002; 13: 623-26.

7. Arif F, Fayyaz J, Hamid A. Awareness among parents of children with thalassemia major. J Pak Med Assoc. 2008 Nov;58(11):621-4. PMID: 19024134.

8. Zaheer HA, Waheed U, Abdella YE, Konings $F$. Thalassemia in Pakistan: A Forward-looking solution to a serious health issue.Glob J Transfus Med 2020;5:108-10
9. Singh J, Singh N, Kumar A, Kedia NB, Agarwal A. Dental and Periodontal Health Status of Beta Thalassemia Major and Sickle Cell Anemic Patients: A Comparative Study. J Int Oral Health 2013; 5(5):53-8

10. IBM Corp. IBM SPSS Statistics for Windows [Internet]. Armonk, NY: IBM Corp; 2019. Available from: https://hadoop.apache.org

11. Qureshi A, Chaudhry S, Shad MA , Izhar F, Khan AA, Is oral health status of children with beta-Thalassemia worse than that of their normal counterparts?, J. Khyber Coll. Dent. 2010; 1 (1): 4-7

12. Phrai-in N, Noikeaw J, Sukprasert N, Taya T, Samnieng P. Oral Health Status and Impact on Oral Health-Related Quality of Life in Children with Thalassemia Major. UIP HEALTH MED. 2017;1

13. Fadel HT, Zolaly MA, Alharbi MO, Qarah LA, Alrehili MS, Alamri AD Tarawah AM. Oral Health Profiles and Related Quality of Life in Thalassemia Children in Relation to Iron Overload: A Cross-Sectional Study. Int J Environ Res Public Health. 2020 Dec 16;17(24):9444. doi: 10.3390/ijerph17249444. PMID: 33339305; PMCID: PMC7766577.

14. Singh J, Singh N, Kumar A, Kedia NB, Agarwal A. Dental and periodontal health status of Beta thalassemia major and sickle cell anemic patients: a comparative study. J Int Oral Health. 2013 Oct;5(5):53-8. Epub 2013 Oct 26. PMID: 24324305; PMCID: PMC3845285.

15. Costacurta M, Epis M, Docimo R. Evaluation of DMFT in paediatric patients with social vulnerability conditions. Eur J Paediatr Dent. 2020 Mar;21(1):70-73. doi: 10.23804/ejpd.2020.21.01.14. PMID: 32183533. 